\title{
MicrocRÉdito \\ Historia y EXPERIENCIAS EXITOSAS DE SU implementación en América Latina
}

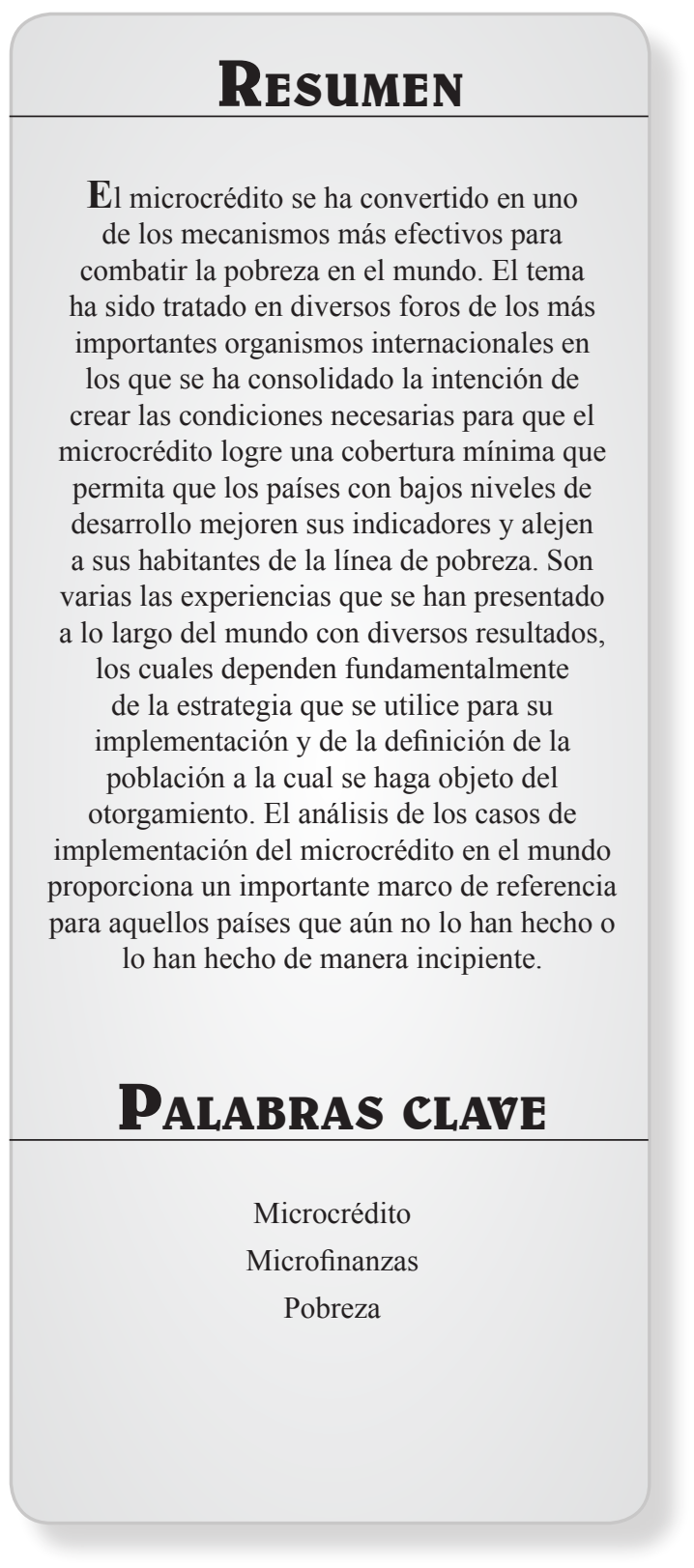

Omar Alonso Patiño*

INTRODUCCIÓN

$\mathbf{L}$ surgen como producto de la necesidad de clientes que no han sido objetivo comercial de los bancos, debido a que no reúnen las condiciones exigidas por los mismos para ser parte de su portafolio de clientes. Esto se debe a que no representan posibilidades ciertas de generar beneficios económicos y por el contrario, se pueden constituir en clientes con altos riesgos en la recuperación de cartera, además de ocasionar altos costos de operación.

\footnotetext{
* Periodista y Administrador de Empresas de la Universidad Pontificia Javeriana, especialista en Gestión de la Calidad y Proceso de Innovación de la Universidad Politécnica de Madrid, Magister en Gestión de Organizaciones de la Universidad de Quebec a Chicoutimi, Candidato a $\mathrm{Ph}$. D. en Ciencias Empresariales de la Universidad de Antonio de Nebrija. Con amplia experiencia en el sector financiero, actualmente se desempeña como Director del Programa de Administración de Empresas de la Universidad EAN.

Para la realización de este artículo se contó con la colaboración de los estudiantes Camilo Cupitre del programa de Administración de Empresas y Laura Natalia Caicedo del programa de Lenguas Modernas de la Universidad EAN, como asistentes de investigación.
}

Este artículo fue entregado el 15 de mayo de 2008 y su publicación aprobada por el Comité Editorial el 18 de junio de 2008. 
El tema del microcrédito ha sido motivo de discusión durante los últimos 25 años en diversos eventos y foros de política, finanzas y economía alrededor del mundo. En la Cumbre del Milenio ${ }^{1}$ celebrada en septiembre de 2000, los estados miembros de las Naciones Unidas, con el propósito de mejorar la calidad de vida de todos los habitantes del mundo, formularon ocho metas que deben cumplirse para el año 2015. Entre estas, también Ilamadas "Objetivos de Desarrollo del Milenio" se destaca la primera que tiene como fin erradicar la pobreza extrema y el hambre. Para esto, se pretende crear puestos de trabajo y desarrollar la agricultura, siendo una alternativa para lograrlo, la creación de mecanismos de acceso a la financiación a la población más pobre del planeta. Estos mecanismos son:

- Programas sostenibles de microcrédito individual y colectivo.

- Programas de estímulos tributarios y financieros al crédito para la población más pobre.

- Programas de ahorro diseñados y destinados a los más pobres.

- Políticas de venta a crédito de bienes básicos a los pobres.

Los primeros indicios de microfinanzas se desarrollaron en Europa hacia 1462, cuando un monje italiano creó la primera casa de empeño oficial con el fin de contrarrestar las prácticas de usura de la época. En 1515, el Papa León $X$ autorizó a las casas de empeño a cobrar intereses con el objetivo de cubrir los costos de operación de la Iglesia Católica.

\footnotetext{
1 Nota de contenido. También conocida como Asamblea del Milenio de las Naciones Unidas, nombre dado al quincuagésimo periodo de sesiones de la Organización de Naciones Unidas que tuvo lugar del 6 al 8 de septiembre de 2000 en conmemoración al cambio de milenio.
}

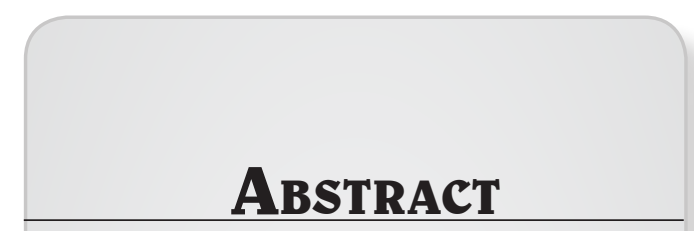

Microcredit has become one of the most effective mechanisms to fight poorness in the world. This topic has been discussed in world events in which different organizations have evidenced the intention to build up the necessary conditions for microcredit to achieve a very small coverage allowing underdeveloped and developing countries to improve indicators and help people abandon their poor economic condition. There have been lots of experiences around the world which really show different results, depending on the strategy used for the implementation of such a program and the definition of population taken for this study. The careful analysis on different world cases dealing with the implementation of a microcredit system provides a referential framework for those countries which haven't adopted this program yet or which have already started it.

\section{KEy WORDS}

\author{
Microcredit \\ Microfinances \\ Poorness
}


En el siglo XVII, el irlandés Jonathan Swift creó el Irish Loan Fund System (Sistema Irlandés de Fondo de Préstamos) el cual proporcionaba pequeños préstamos a los agricultores pobres que no poseían ninguna garantía o aval. En su mejor momento alcanzó hasta el $20 \%$ de todos los hogares irlandeses.

El alemán Friedrich Wilhelm Raiffeisen, junto con otros colaboradores, desarrolló el concepto de cooperativa financiera en el siglo XIX. Este tipo de cooperativa tenía como propósito romper la dependencia de la población rural hacia los prestamistas y mejorar su calidad de vida. Esta se difundió rápidamente en Alemania y otros países de Europa y América del Norte, y posteriormente a los países en vías de desarrollo alrededor del mundo.

Al finalizar la Segunda Guerra Mundial, en Europa Occidental, surgió la necesidad de buscar financiación para reconstruir su infraestructura y capitalizar sus economías. Por esto, las primeras medidas fueron dirigidas al sector agrícola, otorgándoles subsidios de crédito a pequeños granjeros para que realizaran la inversión necesaria y así incrementaran su producción. Estos subsidios no tuvieron gran éxito debido a que las instituciones no fueron capaces de sostenerse con las tasas de interés aplicadas y a que los granjeros no cumplían con sus pagos, pues creían que los subsidios eran regalos del gobierno.

La minimización del riesgo -método tradicional de las instituciones financieras formalesse convirtió en la variable que dificultó el acceso al crédito a la población de más bajos ingresos. Ellos no estaban en la capacidad de aportar los documentos y garantías necesarias para cubrir sus préstamos. La necesidad inmediata del crédito frente al monto, tiempo y esfuerzo requerido para completar el proceso de solicitud, lo hacían inapropiado.

En los comienzos del siglo XX, llegan a las zonas rurales de América Latina los conceptos de ahorro y crédito, los cuales proponían modernizar el sector agrícola, volver productivos los ahorros inactivos, aumentar la inversión a través de créditos y combatir la opresión feudal que se generaba a través del endeudamiento. Estos sistemas de ahorro y crédito fueron ofrecidos por los bancos creados en las grandes urbes de América Latina, bancos de carácter privado o controlados por agencias gubernamentales, diferentes de los europeos, cuyos dueños eran sus propios clientes.

Sin embargo, con la aparición de estos conceptos no se logró el objetivo de llevar los créditos a las poblaciones más vulnerables, a pesar de que la estandarización del otorgamiento y seguimiento (cobro) del crédito hizo posible que las instituciones financieras recortaran una parte de sus gastos administrativos y así pudieran ofrecer una tasa de interés más baja, manteniendo sus márgenes de ganancia.

Para Coulter y Shepherd (2000), la solución para quienes no alcanzaban el crédito era recurrir a los prestamistas informales, los cuales efectuaban sus colocaciones a altas tasas de interés (entre $5 \%$ y $30 \%$ semanal), y ofrecían flexibilidad en los programas de pago, coincidentes con las posibilidades de los solicitantes. Para este sector de la población resultaba más atractivo alcanzar un crédito costoso que no tener acceso a los recursos. Esta respuesta a sus necesidades 
no permitió la reinversión de capital, haciendo que sus utilidades fueran cada vez menores o en muchos casos no se presentaran.

Hacia 1970, a nivel mundial se presenta por primera vez el fenómeno de la migración de la población rural hacia las grandes urbes, hecho motivado por la excesiva oferta de mano de obra no calificada, que superaba la escasa demanda de trabajo en este mercado. El primer problema que se encuentra esta población en las ciudades es el panorama descrito anteriormente.

La dificultad para acceder a un trabajo formal, obligó a las personas a la búsqueda de nuevos mecanismos de subsistencia, con lo cual se empezaron a gestar nuevas actividades tales como la creación de pequeños negocios, llegando a convertirse en lo que actualmente conocemos como microempresarios o microemprendedores.

Para llegar a la noción actual del microcrédito (otorgamiento de créditos a personas que no son atendidas por los grandes bancos), el concepto ha tenido un proceso de evolución, en el que se diferencian las siguientes etapas:

\section{- Microcrédito}

De acuerdo con lo desarrollado en la Conferencialnternacionalsobre Microcrédito ${ }^{2}$ en Washington, DC, el microcrédito consiste en programas de concesión de pequeños créditos a los individuos que alcanzan los más altos índices de pobreza, para que éstos puedan poner en marcha pequeños negocios que generen ingresos para mejorar su nivel de vida y el de sus familias.

\section{- Microfinanzas}

Según Garzón (1996), las microfinanzas son la intermediación financiera a nivel local, (i.e.) en las microfinanzas se incluyen además de créditos, los ahorros, los depósitos y otros servicios financieros. Es un procedimiento local, utilizado por instituciones que acopian recursos y los reasignan regionalmente.

\section{- Las microfinanzas como un sistema financiero incluyente}

En esta última etapa, se define como un sistema financiero auto sostenible y coordinado entre los gobiernos y el sector privado, con el cual se logra llegar con eficiencia a las poblaciones más vulnerables.

Actualmente el microcrédito tiene varias definiciones, algunas de carácter general como la entregada en el marco del Año Internacional del Microcrédito 2005 que lo describe así: "El microcrédito es una pequeña cantidad de dinero en préstamo a un cliente por un banco u otra institución."

\footnotetext{
2 Nota de contenido. La Cumbre de Microcrédito se celebró del 2 al 4 de febrero de 1997. En la actividad se reunieron más de 2.900 personas de 137 países en Washington, DC. Fue el encuentro organizado por una ONG más grande que jamás se haya celebrado, y contó con la presencia tanto de líderes de la industria microfinanciera como Jefes de Estado. Esta Cumbre lanzó una Campaña de nueve años con la meta definida de "trabajar para asegurar que 100 millones de las familias más pobres del mundo, especialmente las mujeres de esas familias, estén recibiendo crédito para autoempleo y otros servicios financieros y de negocio para fines del año 2005."
} 
Para Acción Internacional, una de las instituciones financieras más importantes en el mundo, el microcrédito se define como "Una parte del campo de las microfinanzas, el microcrédito es la prestación de servicios de crédito para los empresarios de bajos ingresos."

En particular, La Asociación Bancaria y de Entidades Financieras de Colombia, Asobancaria (2003), define el microcrédito como "El conjunto de operaciones activas de crédito otorgadas a microempresas cuyo saldo de endeudamiento con la respectiva entidad no supere veinticinco (25) salarios mínimos legales mensuales vigentes."

Según el artículo 39 de la Ley 590 de 2000 para Mipymes (Micro, pequeñas y medianas empresas), el microcrédito se define como "...el sistema de financiamiento a microempresas, dentro del cual el monto máximo por operación de préstamo es de veinticinco (25) salarios mínimos mensuales legales vigentes sin que, en ningún tiempo, el saldo para un sólo deudor pueda sobrepasar dicha cuantía."

En la fijación de políticas de desarrollo para la población mundial, la Organización de Naciones Unidas (ONU) ha establecido como uno de sus más importantes objetivos la reducción de la pobreza y reconoce como uno de los elementos que puede facilitar su cumplimiento, el otorgamiento masivo de operaciones de microcrédito, motivo por el cual declaró el año 2005 como el Año Internacional del Microcrédito. Sin embargo, para que las microfinanzas puedan convertirse en el mecanismo que ayude en el cumplimiento los Objetivos de Desarrollo del Milenio deben afrontar en los próximos años los siguientes retos (OIT, 2005).
- Acceso a los más pobres: ya que en muchas zonas del mundo se está registrando la situación que las IMF, por diferentes factores, no pueden llegar a las poblaciones más pobres o a las más apartadas.

- Mejoramiento en el desempeño de las IMF: debido a que la mayoría de las IMF no han podido hacerse financiera y operativamente rentables, por lo cual no tienen garantizada su permanencia hacia el futuro.

- Conocimiento del impacto socioeconómico de las microfinanzas: si bien se tienen datos sobre la operación y los aspectos técnicos de las microfinanzas a nivel mundial, no sucede lo mismo sobre su impacto en la disminución de la pobreza para las comunidades donde éstas se encuentran presentes.

- Cluster financiero: puesto que se hace necesario formar unidades productivas conjuntas que faciliten el desarrollo conjunto de todos los actores de las microfinanzas, mediante su interacción y colaboración en ambientes propicios para su crecimiento y desarrollo.

\section{- Promoción y apoyo de los mercados} de capitales internacionales hacia Ias IMF: porque hasta ahora se ha dependido excesivamente de los recursos gubernamentales y de las Entidades Multilaterales, los cuales son limitados, y ante el panorama actual donde cada vez más IMF logran ser financieramente rentables, se ve la oportunidad de acudir a los mercados de capitales, los cuales podrían inyectar los recursos que las microfinanzas necesitan 
para crecer al ritmo deseado e incrementar su oferta y cobertura.

- Mejora de las políticas de fomento hacia la financiación social. Porque actualmente en muchos países la normatividad financiera se enfoca en las operaciones de la banca tradicional y obliga a las IMF a competir bajo las mismas condiciones, lo cual constituye un elemento disuasivo para la inversión en financiación que tenga una función social que no produce tantos beneficios (o no produce ningún beneficio en algunos casos) como una inversión en otros tipos de financiación.

\section{IMPACTO DEL MiCROFINANCIAMIENTO(CGAP, 2003)}

Ee entiende como impacto del microfinanciamiento, el efecto que tiene en la vida de los pobres y sus familias los servicios financieros.

Los indicadores del impacto del microcrédito que generalmente se utilizan (crecimiento del ingreso, generación de activos, generación de empleo, reducción de la vulnerabilidad, etc.) sólo evalúan el efecto que tienen los créditos sobre los pobres. Pero la pobreza es un término más amplio que involucra un número mayor de variables tales como el mejoramiento de condiciones en los servicios de salud y educación, por ejemplo. El microcrédito, como está concebido, debe generar efectos sobre otras variables tales como la situación de la mujer cabeza de familia, el aumento en sus ingresos, el mejoramiento de su autoestima y el control que tiene sobre los ingresos de la familia.

Así las cosas, las mediciones de los impactos del microcrédito deben hacerse sobre un número amplio de variables entre las cuales se combinen las expresadas anteriormente con otras que pueden depender de las condiciones particulares de cada una de las áreas en las cuales se implemente y los objetivos que se persiguen con su otorgamiento o con los posibles efectos que pueda generar sobre la población objetivo o sobre las condiciones económicas del país. En un marco mucho más amplio, la medición debe correlacionarse con indicadores de pobreza.

Los impactos que pueda generar el microcrédito no sólo dependen de las variables anteriormente mencionadas. Existen algunas que son imputables a cada uno de los actores participantes en su cadena. En el caso de los beneficiarios, las condiciones de uso de los recursos que les otorgan son fundamentales para establecer el efecto que sobre sus condiciones de vida puedan llegar a tener. El destino de la inversión, las capacidades para el manejo de la misma, el conocimiento del negocio o la actividad económica para la cual se solicitan los recursos, así como la calidad del acceso a servicios de salud y educación, son factores que influyen decididamente sobre los niveles de impacto. 
En el caso de las instituciones financieras o de los intermediarios otorgantes del crédito, su capacidad para llegar a la población que requiere de sus servicios, flexibilizar los mecanismos de otorgamiento de operaciones crediticias y generar esquemas de continuidad que les permitan ser competitivos frente a los ofertantes de servicios para otros públicos, es determinante en el momento de establecer las posibilidades de impactar sobre la población más pobre.

Además, el éxito o fracaso del microfinanciamiento está atado a múltiples factores externos como el entorno macroeconómico, la sostenibilidad del negocio a largo plazo, inestabilidad y/o ineficiencia en el marco jurídico, corrupción e inseguridad social, los desastres naturales, entre otros que afectan significativamente el impacto de los servicios financieros.

Según el CGAP (Consultative Group to Assist the Poor), el impacto del microfinanciamiento se puede observar en tres niveles:

\section{$\checkmark$ Doméstico}

- Aumento del ingreso familiar (diversificación de fuentes de ingresos o crecimiento de empresas).

- Mejora y/o cambio en la combinación de activos (tierras, mejoras de viviendas, animales y bienes de consumo duraderos, activos humanos).

- Mejora en la gestión del riesgo y aprovechamiento de oportunidades.

\section{$\checkmark$ Individual}

- Empoderamiento específicamente en las mujeres.

- Capacidad de ahorro.

\section{$\checkmark$ Empresarial}

- Aumento del ingreso de las empresas.

- Generación de empleo.

A pesar de los distintos aspectos sobre los cuales se considera que el microcrédito tiene efectos, no se puede pensar que llegue a sustituir otras políticas estatales de inversión social, por el contrario, puede ser un complemento importante de ellas. El microfinanciamiento es apenas una de las políticas que deben ser implementadas por los gobiernos de los países en desarrollo, tal como lo manifiesta Cohen (2007):

"Afortunadamente, las evaluaciones de los efectos sociales han demostrado que los servicios financieros a los pobres hacen que mejoren sus vidas al aumentar sus ingresos y mejorar su capacidad de pagar los servicios sociales. No obstante, aunque el microfinanciamiento es un importante catalizador en la lucha contra la pobreza, no es un remedio mágico. Escapar a la pobreza suele ser un proceso lento y desigual.

El financiamiento a cargo de los donantes para fines de microfinanciamiento no puede sustituir, sino complementar, las inversiones en servicios esenciales como salud, educación e infraestructuras."

(CGAP, 2003) 


\section{Microfinanzas y La OIT: GENERACión dE EMPLEO (OIT, 2005)}

"Incumbe a la Organización Internacional del

Trabajo examinar y considerar, las decisiones $y$ políticas internacionales de carácter económico y financiero bajo el prisma de la justicia social" Constitución de la OIT

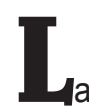

organización Mundial del Trabajo, OIT, considera que las microfi-nanzas tiene una labor importante ligada a los programas de desarrollo de la OIT, específicamente aquellos relacionados con los principios fundamentales de trabajo (abolición del trabajo forzoso y del trabajo infantil).

En este sentido, la OIT vincula a las microfinanzas con el trabajo decente lo cual supone que mujeres y hombres tengan la oportunidad de tener un empleo digno y productivo ya que estos proporcionan oportunidades para pequeñas inversiones en autoempleo y en la creación de puestos de trabajo.

Una adecuada política entre microfinanzas y trabajo decente traen consigo:

- Que los niños no tengan que trabajar y por ende abandonar el sistema educativo permitiendo que sus padres generen los ingresos necesarios.
- Que los empleados obtengan una alternativa diferente de financiación a la de sus empleadores, evita que éstos puedan llevar a cabo acciones de servidumbre a cambio del pago de la deuda.

- Que las mujeres puedan acceder a iniciativas laborales reduciendo la posibilidad de ser objeto del tráfico de seres humanos.

Siendo las microfinanzas una parte fundamental en el cumplimiento de los objetivos de la OIT, este organismo crea en 1991 el programa de Finanzas Sociales como un programa fundamental en el análisis, la evaluación y la difusión a cuestiones referentes al sector financiero que sean notables para el empleo y la justicia social. El programa enfoca sus investigaciones y estudios en la evaluación y estudio de tres resultados que considera muy importantes: reducción de la vulnerabilidad, inversión en creación de empleo, y acciones para hacer que las políticas financieras estén más enfocadas hacia empleo. 


\section{AnÁlisis de casos E implementación de metodologías en América Latina}

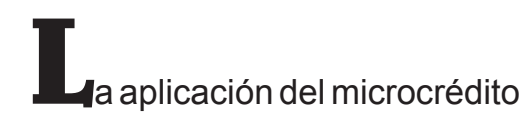
como parte de las políticas de combate a las condiciones de pobreza es un fenómeno de reciente suceso, a pesar de ser un mecanismo que cuenta con cerca de cuarenta años de existencia. A continuación se presentan algunos de los casos de implementación más reconocidos o con indicadores de éxito sobresalientes.

\subsection{Bolivia}

Este país cuenta con una de las instituciones microfinancieras más reconocidas a nivel latinoamericano y mundial. Se trata del Banco Solidario S.A. (Bancosol), banco comercial privado, con todas las ventajas que ofrece un banco comercial y sujeto a todas las regulaciones como cualquier otro banco de su país, pero con origen y enfoque comercial distinto del de sus competidores.

Bancosol tuvo su origen en la Fundación para la Promoción y Desarrollo de la Microempresa, PRODEM, que inició operaciones en 1987 como una ONG dedicada a proveer créditos a pequeños proyectos empresariales de personas con escasos recursos. Entre el año de su fundación y 1992, PRODEM tuvo un desempeño más que aceptable que le permitió llegar a ser una organización financieramente sostenible, alcanzando los US $\$ 19$ millones en desembolsos
(Gonzalez, Schreiner, Rodríguez y Navas, 1996). No obstante, para 1990 PRODEM sintió la necesidad de expandirse, ya que no estaba creciendo a la medida que lo hacia la demanda de los créditos, además, al ser una ONG, se encontraba legalmente restringida para ofrecer otros servicios financieros más allá del crédito y para acceder a fuentes de fondos más flexibles que los de las instituciones donantes, los cuales no le permitirían a esta organización responder a sustanciales demandas de crédito fácilmente identificables y manejar sus flujos de fondos más eficientemente. Por esa razón decidieron iniciar un proceso de transición para poder convertirse en un banco comercial ya que al poder recibir recursos de los depósitos y de otras fuentes se podía abrir el mercado para el microcrédito e independizarse de las donaciones.

Este proceso de transición comenzó con un requerimiento para todos los clientes del crédito de depositar el $5 \%$ del crédito como ahorro, continuó durante los dos años siguientes con los estudios técnicos y de factibilidad, con la promoción de este proceso entre potenciales inversionistas nacionales e internacionales y con el traspaso de fondos de la ONG al banco con los donantes y por último con el diseño y coordinación de nuevas operaciones y planes para el banco y para la ONG. Así, después de haber obtenido las aprobaciones legales en 1992, se dio inicio a BancoSol que recibió de PRODEM una cartera de préstamos de US\$ 3,960,000 con aproximadamente 14,300 clientes activos, 
bienes raíces de sus sucursales urbanas y un pasivo subsidiado proveniente de un préstamo por US\$ 850,000 al ocho por ciento anual, (Gonzalez, Schreiner, Rodríguez y Navas, 1996). además de una serie de activos intangibles como la plataforma tecnológica, un know how en otorgamiento de crédito, una reputación de marca y otros, lo que le dio derecho a poseer el $44.2 \%$ de las acciones del nuevo banco, el resto del financiamiento fue cubierto por diversos inversionistas privados locales y extranjeros ${ }^{3}$; por su parte PRODEM continúo con sus propias operaciones financieras, especializándose en microfinanzas rurales en contraposición a su interés original en las finanzas urbanas.

Durante sus primeros años de operación BancoSol tuvo resultados realmente sobresalientes. Desde principios de 1992 hasta finales de 1994, la cartera de BancoSol se incrementó diez veces en términos reales. (Gonzalez, Schreiner, Rodríguez y Navas, 1996). Para este último año, su número de clientes activos había sobrepasado los 60.000. Ninguna otra organización de microfinanzas en Latinoamérica tenía en ese momento un número tan elevado de clientes en su cartera. Para 1995, el banco entró en un proceso de estabilización de sus resultados y comenzó a verse que, si bien eran excelentes en números, tenían algunos reparos en cuanto al impacto de la profundidad de sus políticas en la población, para empezar: si bien los clientes de BancoSol son pobres, no están entre los más pobres de los bolivianos (Navajas et al., 1996). Igualmente, parte de los socios, se criticaba por que el crecimiento del saldo promedio de los préstamos vigentes (de US\$ 300 en los primeros días de BancoSol hasta más de US\$ 600 a comienzos de 1994) no tuviera la misma proporción que las cifras de número de clientes mencionadas anteriormente, este monto promedio apenas superaba la mitad del PIB per cápita de Bolivia para ese año, y el establecimiento de requisitos como tener una actividad productiva que debía estar operando por lo menos un año para poder solicitar un préstamo. Estas condiciones se han mantenido durante los años siguientes, reduciendo los porcentajes de crecimiento del banco en cuanto a los montos y al número de créditos desembolsados, como lo podemos ver en la siguiente tabla que muestra los resultados de BancoSol en sus primeros 10 años de operación.

\footnotetext{
3 Nota de contenido: Los accionistas internacionales incluyen a Acción Internacional, la Fundación Calmeadow, la Societé D'Investissement et de Developpement International, la Fundación Rockefeller y la Corporación Inter-Americana de Inversiones. Entre los accionista nacionales originalmente estaban una ONG (PRODEM) y tres bancos privados, tres empresas y cinco individuos prominentes, incluyendo al Presidente de la República, Sánchez de Losada.
} 
Tabla 1

Logros alcanzados por BancoSol

\begin{tabular}{|l|c|c|c|}
\hline Año & $\begin{array}{c}\text { Préstamos Anuales } \\
\text { Desembolsados (US \$) }\end{array}$ & $\begin{array}{c}\text { Acumulado } \\
\text { Desembolsado (US \$) }\end{array}$ & $\begin{array}{c}\mathbf{N}^{\mathbf{0}} \text { de Microempresas Proyectos } \\
\text { Financiados }\end{array}$ \\
\hline 1994 & & 147,193 & 294.659 \\
\hline 1995 & 83.327 & 230.520 & 432.892 \\
\hline 1996 & 103.788 & 334.308 & 630.667 \\
\hline 1997 & 112.228 & 446.537 & 775.899 \\
\hline 1998 & 136.614 & 583.150 & 911.193 \\
\hline 1999 & 122.535 & 705.685 & 948.747 \\
\hline 2000 & 80.828 & 786.513 & 979.893 \\
\hline 2001 & 41.734 & 828.247 & 994.469 \\
\hline 2002 & 87.378 & 915.625 & 1 '009.045 \\
\hline
\end{tabular}

Fuente. www.bancosol.com.bo

Sin embargo, y a pesar de todos los problemas y críticas mencionadas BancoSol se ha mantenido como una entidad financieramente estable y ha trascendido las fronteras de su país como un hito y un ejemplo para las entidades microfinancieras, ya que fue la primera entidad de este tipo en el mundo que pasó de ser una ONG a un banco comercial.

Tabla 2

Evolución de la cartera y número de clientes de BancoSol entre 2003 Y 2006

\begin{tabular}{|l|l|l|}
\hline \multicolumn{1}{|c|}{ Año } & \multicolumn{1}{|c|}{ Cartera USd (Miles) } & Número de Clientes Activos \\
\hline 2003 & 91.175 & 56.707 \\
\hline 2004 & 108.560 & 71.609 \\
\hline 2005 & 130.106 & 85.000 \\
\hline 2006 & 165.155 & 103.786 \\
\hline
\end{tabular}

Fuente. Anuarios BancoSol, 2003, 2004, 2005 y 2006. http://www.bancosol.com.bo/sp/memorias.html

\subsection{Nicaragua}

Este país centroamericano que ha sido testigo de múltiples revoluciones y guerras civiles (la última de ellas terminó con la firma de los acuerdos de paz de 1988) cuenta con exportaciones totales que suman alrededor de 300 millones de dólares para 2006, de los cuales el $60 \%$ corresponden a la agricultura.
Además de esto, su PIB es de 5370 millones de dólares a 2006 con un crecimiento del $3,7 \%$ respecto al año anterior, un ingreso per capita de US \$994 y una inflación del $9,47 \%$ para el mismo año (US Department of State, 2008). Siendo considerado por el FMI y otras instituciones en el año 2005 la 
segunda economía más pobre de América Latina (sólo superando a Haiti) (Martínez, 2005), ha tenido a partir de los años 80 múltiples iniciativas de microcrédito enfocadas principalmente en la actividad rural y en las mujeres. Sin embargo, estos esfuerzos tanto públicos como privados obedecen a políticas dispersas e incoherentes entre si (Karremans y Petra, 2003). Como prueba de esto, esta la escasa documentación acerca de microfinanzas disponible para este país.

En el campo de las microfinanzas, para 2002 la demanda de microcrédito alcanzaba los US $\$ 70.200 .000$ para Pymes, US $\$ 20.000 .000$ paraloscréditospersonalesyUS $\$ 207.900 .000$ para el sector rural. No obstante, la cartera de las entidades microfinancieras era apenas de US $\$ 126.500 .000$, y su máximo potencial de recursos era de US \$181.300.000 (Karremans y Petra, 2003), por lo cual había una gran cantidad de demanda insatisfecha. Esto se debe a que el tamaño de la oferta de microcrédito no era la adecuada, siendo el mayor prestatario el Banco Centroamericano de Integración Económica, con una cartera de US \$100.000.000 (2002) (Karremans y Petra, 2003). Este banco, creado en 1960 por los gobiernos de Guatemala, El Salvador, Honduras, Nicaragua y Costa Rica y que posteriormente recibió las adhesiones de China, Argentina, Colombia, y España, con el fin de promover el desarrollo social en la región centroamericana, para 2005 en Nicaragua, destinaba sus aportes principalmente al combate de la pobreza, como podemos ver en la figura con 63,7 millones de dólares, en segundo lugar está el apoyo a la inserción en la globalización de las Pymes con 34,9 millones de dólares, y por último, está el apoyo a la integración regional de las Pymes con 16,5 millones de dólares, para un total de cartera a 2005 de 115,2 millones de dólares.

Figura 1

Desembolsos otorgados por el BCIE en el periodo 2005 por país (Cifras en Millones de dólares)

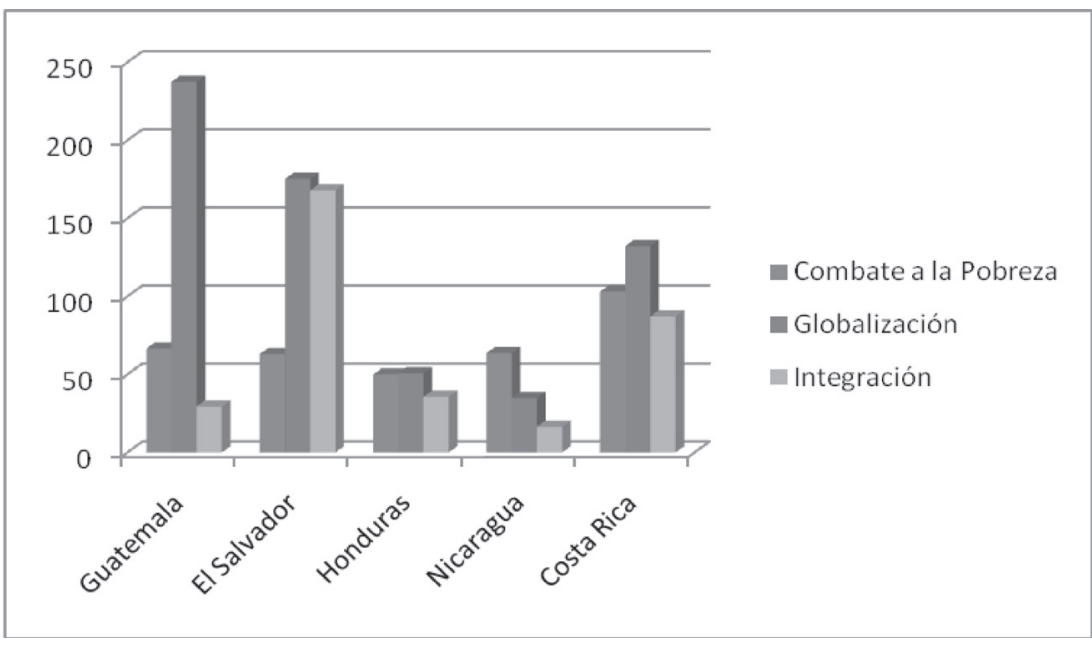

Fuente. El impacto social del microcrédito en Nicaragua, percepciones de prestatarios y prestatarias de siete microfinancieras, Sonia Agurto Vilches, Maria Alejandra Guido, Fundación Internacional para el Desafío Económico Global (FIDEG) y el Consejo Coordinador De Wisconsin con Nicaragua, Managua Enero de 2006. 


\subsection{Guatemala}

La actividad microfinanciera en su forma actual surge en Guatemala en los años ochenta, promovida en parte por el Programa Nacional de la Microempresa. En esa época, la estrategia era coordinar los esfuerzos del Estado con los de las ONG para ejecutar programas que contemplaban una mezcla de capacitación, crédito y asistencia técnica dirigida a los microempresarios. El enfoque se orientaba a fortalecer actividades de manufactura y servicios, por considerar que estas generan valor agregado y empleo, dejando de lado las actividades de comercio para el apoyo financiero.

Paralela a esta iniciativa, entra al país, en la segunda mitad de la década, el programa de la ONG estadounidense Acción Internacional y se inicia con una estrategia agresiva de expansión. Esta ONG implementa sus acciones por medio de dos ONG, Génesis Empresarial en la capital de Guatemala y FUNDAP en el Occidente. Por cambio de estrategia del Gobierno, en 1991 se limita el apoyo a este sector y los fondos se orientan entonces hacia el apoyo de la banca privada, creando un fideicomiso en el Banco de Guatemala para que la banca privada pudiera canalizar los recursos en el primer piso.

En el transcurso de los años noventa, Guatemala experimenta un gran número de iniciativas en el área de microfinanzas, ya no sólo propiciadas por organismos de origen norteamericano sino también y en creciente medida, por iniciativas de origen y con fondos europeos. En esta categoría se presentan, por ejemplo, iniciativas de la Cooperación Belga, seguidas por los programas de desarrollo rural de la Unión Europea en los departamentos considerados marginales del país.

Asíresultaqueelsectordelas microfinanzasen Guatemala ha experimentado cambios profundos, mostrando transformación, especialización y a la vez la búsqueda de estándares de eficiencia y productividad. El dinamismo observado en los últimos años se ha gestado a raíz de los siguientes acontecimientos:

- Firma de los Acuerdos de Paz. En diciembre de 1996, se firman en Guatemala estos acuerdos. Esto trae como consecuencia un cambio de las estrategias de apoyo al sector de organizaciones de desarrollo, los que se resumen en dos aspectos: a) Se fortalece la canalización de recursos por medio del sector gubernamental; y b) Se deja - al menos en papel - el enfoque "asistencialista" para remplazarlo con otro basado en la sostenibilidad, lo que crea las condiciones para iniciar un proceso de transformación del sector microfinanciero.

- Crisis del sector financiero formal. Esta se manifiesta en parte, por la quiebra de instituciones financieras privadas (bancos comerciales y sociedades privadas) y por otra parte también por el receso de la banca estatal de fomento. Un reflejo es la transformación deBANDESA ${ }^{4}$ enBANRURAL ${ }^{5}$, proceso que contó con un fuerte apoyo de la cooperación, especialmente la AID (apoyo a reformas institucionales), el BID y el BCIE

\footnotetext{
4 Nota de Contenido: BANDESA es el antiguo Banco Nacional de Desarrollo Agrícola S.A.

5 Nota de Contenido: Banco de Desarrollo Rural S. A. (Banrural S. A.).
} 
(reformas institucionales y cooperación con recursos financieros). Otro elemento para contrarrestar la crisis del sector financiero es el programa de Bancos Comunales, implementado en BANCAFE ${ }^{6}$.

- Reformas a la Ley. Se aprueban leyes orientadas a permitir el financiamiento del sector por parte de BANRURAL, entre otros, como instancia de segundo piso, convirtiéndose en fiduciario de gran proporción de los fondos de fideicomisos en Guatemala (entre otros, los de los proyectos de la UE). Por otra parte, la aprobación del Decreto 44-2000, coloca a las organizaciones no reguladas en una posición distinta de las intermediarias formales. Esto crea un ambiente propicio para que las organizaciones de microfinanzas busquen espacios para analizar las implicaciones del nuevo decreto y nuevas modalidades de operar en el futuro.

Las circunstancias señaladas permiten también la creación de la Red de Instituciones de Microfinanzas de Guatemala REDIMIF creada en el 2001. Esta iniciativa genera sus resultados dos años después: estudios sobre la escala y el desempeño de las organizaciones, iniciativas de estándares uniformes de evaluación, propuestas de fortalecimiento del sector, elaboración de una planeación estratégica y operativa para la red y la aprobación de un proyecto para el fortalecimiento institucional.

Otra iniciativa reciente es la creación de la Corporación de Referencias Crediticias, Sociedad Anónima (CREDIREF S.A.), mejor conocida como la Central de Riesgos, como socia con la REDIMIF, BANRURAL y BANCAFE. A través de la Central, los socios comparten información sobre sus carteras y clientes. Su objetivo es reducir niveles de morosidad (mediante intercambio de referencias sobre un total de unos 200.000 clientes) y promover la cultura de pago entre los clientes de las instituciones afiliadas.

Actualmente en Guatemala, la cooperación internacional apoya al sector microfinanciero mediante un comité asesor especializado en microfinanzas, Ilamado Comité de Cooperantes Internacionales para las Microfinanzas (CCMF). Se trata de un subcomité de la Mesa de Coordinación de Cooperantes Internacionales MIPYME Guatemala. Este comité cuenta con una Agenda Estratégica, que contempla las principales acciones a desarrollar y su objetivo es maximizar las inversiones, no duplicar esfuerzos e implementar una estrategia de apoyo integral al mercado de microfinanzas en Guatemala que engloba una cartera total agregada y conocida por el orden de 240 millones de dólares. (Villalobos, Sanders y Ruijter, 2003).

6 Nota de Contenido: Banco del Café S.A. 


\section{Bibliografía}

Acción Internacional.(2007): About our organizations, Key Statistics, ACCION "Micro" Loans \& Impact on World Poverty. www.accion.org/NETCOMMUNITY/Page.aspx?pid=492\&srcid=254

Banco Sol S. A. Bancosol: Form microcredit to microfinance. The Origins of Microfinance Capítulo I, pp. 5-6.

Banco Mundial. Documento Precis. Departamento de Evaluación de Operaciones. Verano de 2002, Número 226

Banco Mundial.(2007): Indicadores de Desarrollo Mundial. http://web.worldbank.org/WBSITE/ EXTERNAL/BANCOMUNDIAL/NEWSSPANISH/0,,contentMDK:21299920 pagePK:6425704 3 piPK:437376 theSitePK:1074568,00.html

Centralamerica.com. Nicaragua. General information. The economy. http://centralamerica.com/nicaragua/info/general.htm\#economy

CGAP. Página Oficial CGAP.

http://www.cgap.org/portal/site/CGAP/menuitem.9a218408ac5bc61fae6c6210591010a0/

CGAP. (2003): El impacto del Microfinanciamiento. Reseñas para agencias de cooperación. No 13, Julio.

Cohen, Monique.(2003): El impacto del microfinanciamiento. Ayudando a mejorar la eficacia de las agencias de cooperación en las microfinanzas. CGAP, julio.

CONDUSEF.(2002): Perspectivas. El Boom de los Bancos de Pobres. No. 33 Diciembre de 2002. http://www.condusef.gob.mx/Revista/2002/33/perspectivas_33.htm

Coulter, Jonathan, y Shepherd, Andrew W. (2007). El crédito prendario: Una metodología para desarrollar los mercados agrícolas. Boletín de servicios agrícolas de la FAO, 120. Roma.

Daley-Harris, Sam.(2007): Informe del Estado de la Campaña de la Cumbre del Microcrédito 2007. Washington D.C. Página 2, 4, 20.

Fernández F, Benito.(2005): El potencial de las microfinanzas para el desarrollo local en las experiencias impulsadas por AeA en la región de América Latina (2000-2003). 
FINCA Internacional. (2007): Finca country programs, regions and countries. Latin America http://www.villagebanking.org/site/c.erKPI2PCloE/b.2591249/k.695B/FINCA_Country_ Programs.htm.

González, Schreiner, Meyer; Rodríguez; Meza y Navajas. (1996): BANCOSOL: El reto del crecimiento en organizaciones de microfinanzas.

Guillamon (2002): FOMIN: Microfinanzas. Oficina de Evaluación y Supervisión, Evaluación de los Proyectos. Banco Interamericano de Desarrollo, Washington D.C., Noviembre.

Karremans, Petry, P. (2003): Mujeres rurales y la brecha entre oferta y demanda de microcréditos: los casos de Costa Rica y Nicaragua. Informe Final de Consultoría para el IICA y el BID. San José, Costa Rica, Agosto.

Martínez, Nicaragua: ¿Y la economía qué? [En línea]. BBC Mundo. 5 de septiembre de 2005. http://news.bbc.co.uk/hi/spanish/business/barometro_economico/newsid_4214000/4214962. stm.

McKenna, Navas y Soriano (2007): Microscopio. El entorno de negocios para las microfinanzas en América Latina, Economist Intelligence Unit (EIU) con el apoyo del Banco Interamericano de Desarrollo (BID) y la Corporación Andina de Fomento (CAF).

MicroRate, Agencia, Codespa, Ceami. Segundo informe. La industria de las microfinanzas en América Latina. 2007

Moslares, Turmo, Ortiz y Reyes G. (2004): La inversión extranjera directa y el comercio internacional. El caso de Bolivia, la UE y España. Boletín Económico de Ice $N^{\circ} 2805$, Mayo.

OIT. (2005): Hechos sobre microfinanzas y trabajo decente.

http://www.ilo.org/wcmsp5/groups/public/---dgreports/---dcomm/documents/publication/ wcms_067569.pdf

Pérez. (1999): La inversión extranjera directa en Cuba. Peculiaridades. http://www.nodo50.org/cubasigloXXI/economia/villanueva2_300602.htm

Roberts (2003): "El microcrédito y su aporte al desarrollo económico", Pontificia Universidad Católica Argentina, Facultad de Ciencias Sociales y Económicas, Departamento de Economía, Buenos Aires, Octubre.

Rutherford (2000): "The poor and their Money". 
Safesave, página oficial. Junio 21 de 2008 http://www.safesave.org/

Schneider (2004): The Size of the Shadow Economies of 145 Countries all over the World: First Results over the Period 1999 to 2003, Universidad de Linz e IZA Bonn, diciembre.

Schumpeter. "Teoría del desenvolvimiento económico". México. MX. Fondo de Cultura Económica. Grandes Estudios, v. 3. 1974. cap 3 pg 168-169).

Schwab Foundation for social entrepreneurship. Campo de Actividad: mujeres, desarrollo rural, microcrédito, condiciones laborales, cultura/artesanía, comunicación/medios, SEWA. 2007 http://www.schwabfound.org/schwabentrepreneurs_sp.htm?schwabid=285).

U.S. Department of State. Bureau of Western Hemisphere Affairs January 2008. Background Note: Nicaragua http://www.state.gov/r/pa/ei/bgn/1850.htm

Villalobos, Sanders (2003): Marieke de Ruijter de Wildt. Microfinanzas en Centroamérica: los avances y desafíos. CEPAL Unidad de Estudios Especiales, Santiago de Chile.

Vonderlack y Schreiner. Mujeres, microfinanzas y ahorro: Lecciones y propuestas Center for Social Development Washington University in St. Louis , Junio 2001

Von Stauffenberg y Pérez M (2005): Informe sobre el estado de las microfinanzas en América Latina, agencia MicroRate, Codespa, Ceami.

Yunus, Muhamad (1998). Hacia un mundo sin pobreza. Madrid: Andrés Bello.

Zapata L. (2007): El Papel de las Microfinanzas en la erradicación de la pobreza. Universidad EAFIT. 


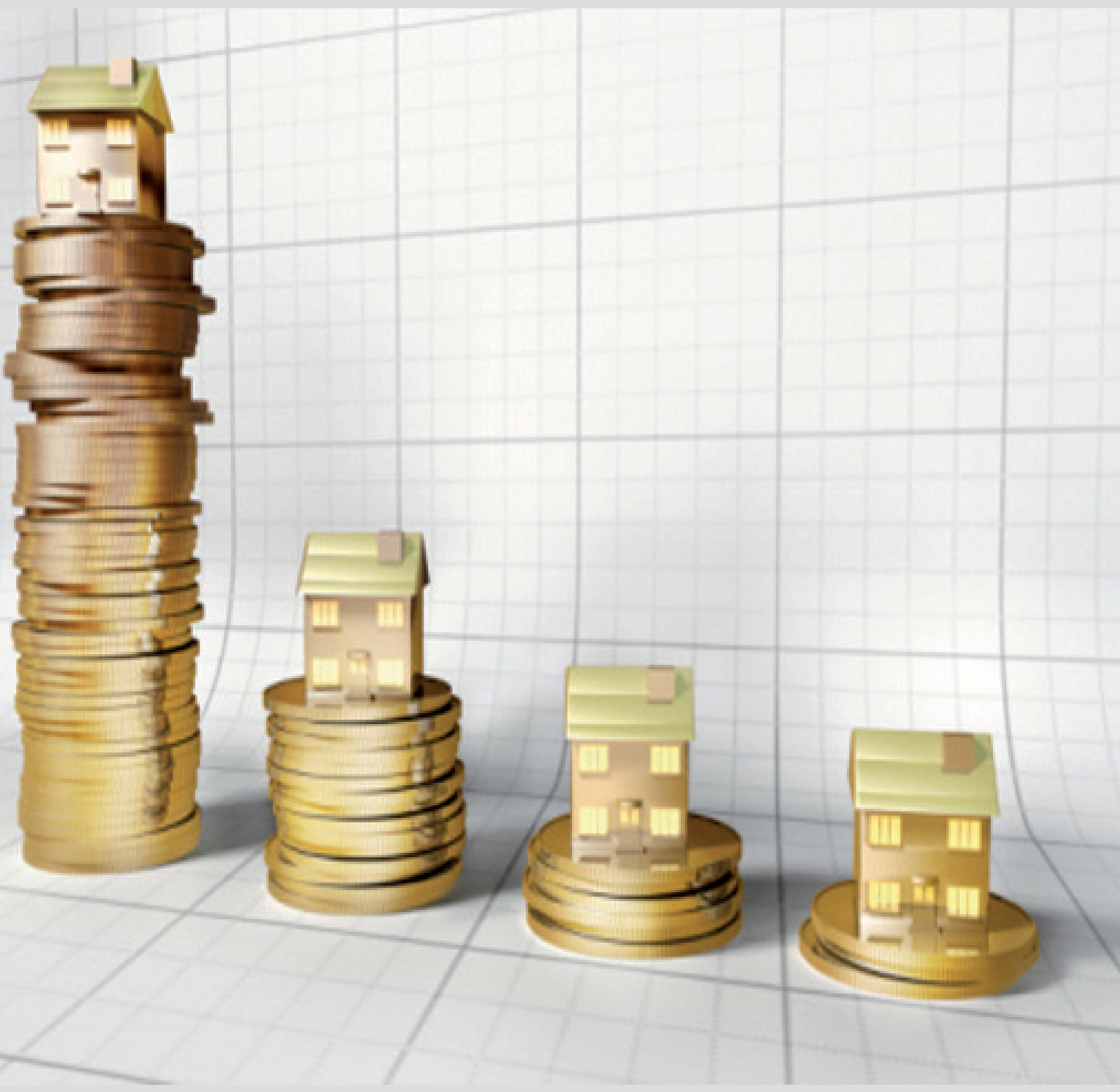

